\title{
BLOOD TRANSFUSIONS FOR SEVERE MALARIA-RELATED ANEMIA IN AFRICA: A DECISION ANALYSIS
}

\author{
CHARLES O. OBONYO, EWOUT W. STEYERBERG, AGGREY J. OLOO, AND J. DIK F. HABBEMA \\ Center for Clinical Decision Sciences, Department of Public Health, Erasmus University Medical School, Rotterdam, The \\ Netherlands; Vector Biology and Control Research Centre, Kenya Medical Research Institute, Kisumu, Kenya
}

\begin{abstract}
Severe childhood malarial anemia is commonly treated using blood transfusion. Although transfusion may decrease short-term mortality, the risk of human immunodeficiency virus (HIV) transmission is considerable in Africa. We constructed a decision tree to weigh the short-term mortality benefit of transfusion against HIV infection risk. Probability estimates were derived from published studies. The base-case was a two-year-old child with a $13.5 \%$ mortality risk to be transfused with screened or unscreened blood ( $1 \%$ or $13 \%$ HIV contamination risk, respectively), with reduction of mortality to $5.5 \%$ by transfusion (odds ratio $=2.7$ ), and a $2.4 \%$ risk of fatal transfusion complications. A sensitivity analysis was performed to assess the influence of variation in these estimates. If a child developed acquired immunodeficiency syndrome, survival was weighed as one-tenth of normal survival. For the base-case, we found that transfusion with screened blood provided a survival benefit of 5\%. In contrast, transfusion with unscreened blood decreased survival by $2 \%$. Patients with a mortality risk $<5 \%$ derived no benefit from a transfusion with screened blood. Other important factors for the benefit of transfusion were the effectiveness of transfusion in reducing mortality and the risk of blood contamination. A blood transfusion was clearly beneficial if the mortality risk was high and the risk of contamination was low. Our findings can be used as a basis for a clinical transfusion policy that limits transfusions to situations in which they are likely to be beneficial. This will in turn optimize child survival and prevent unnecessary exposure of low risk children to the transfusion risks.
\end{abstract}

With the risk of transmission of the human immunodeficiency virus type-1 (HIV-1), the use of blood transfusion in the management of severe pediatric anemia has become an important clinical decision problem in Africa. ${ }^{1,2}$ Severe malaria-associated pediatric anemia increasingly is a major cause of hospital admission and death as the spread of chloroquine-resistant Plasmodium falciparum malaria intensifies across the continent. ${ }^{3-6}$ Subsequently, the demand for blood transfusions has increased. In some settings, $20-50 \%$ of the hospitalized children were transfused. ${ }^{7,8}$

The prevalence of HIV infection among blood donors has increased in most developing countries, and at least $10 \%$ of all African pediatric acquired immunodeficiency syndrome (AIDS) cases may have arisen from contaminated blood transfusions. ${ }^{2,9-11}$ Many African countries, particularly where $P$. falciparum malaria is endemic and HIV/AIDS is a major health issue, cannot maintain an adequate blood supply, and fail to screen all their donated blood. ${ }^{11-14}$ Even screened blood can be infectious, with a risk that depends on the background seroprevalence among the blood donors and on the quality of the screening. ${ }^{12,15}$

The mortality from severe anemia is known to be higher with more intense $P$. falciparum transmission patterns and patient characteristics such as younger age, lower hemoglobin level, and presence of respiratory distress or impaired consciousness..$^{7,16-22}$ A survival benefit of transfusion was found in an observational study in western Kenya, ${ }^{7}$ but the effectiveness of transfusions has unfortunately not been studied in randomized controlled trials.

In this study, we examined when administration of a blood transfusion is beneficial, using decision analysis as a framework for synthesis of the available evidence. ${ }^{23}$ We weigh the risks of mortality with severe anemia against complications and HIV risks associated with receiving a blood transfusion. This understanding will enable clinicians to perform informed risk-benefit evaluations prior to making any transfusion decisions.

\section{METHODS}

Model structure and assumptions. A decision analysis model was constructed for the problem of whether or not to transfuse a severely anemic child less than five years of age with an acute $P$. falciparum malaria infection. Severe malarial anemia was defined as a hemoglobin level $<5 \mathrm{~g} / \mathrm{dL}$ and any level of peripheral malaria parasitemia. We structured the problem similar to a previously published decision tree (Figure 1). ${ }^{24}$

Depending on the clinical presentation, the attending physician in consultation with the accompanying guardian decides on the need for a transfusion. When the strategy "Do not transfuse" is chosen, the patient may die of the pathologic processes associated with anemia and/or malaria, or survive the acute phase of the disease. For transfusion, a blood unit is selected or a donor sought. In the short-term, the recipients may die of anemia, malaria, or non-HIV transfusion complications. The selected blood unit may be HIV contaminated. Even if screened, this status cannot usually be known with certainty, specifically due to laboratory error, limits in test sensitivity, or window period (time between infection and detection of antibodies to HIV). ${ }^{15}$ Surviving patients may acquire HIV infection and develop AIDS. Treatment outcomes considered in this analysis were survival, death, or survival with AIDS.

In structuring this problem, we made a number of assumptions. We assumed that the decision to transfuse means immediate transfusion. In practice, the decision may take the form of either an immediate transfusion or one after a period of patient observation during which the patient's state is monitored for deterioration. Second, the effectiveness of a transfusion is assumed to be equal for patients risking death from anemia and patients risking death from malaria (particularly cerebral malaria). This may be reasonable since most of the risk factors overlap (e.g., impaired consciousness and respiratory distress). ${ }^{16}$ Third, we assumed that the pa- 


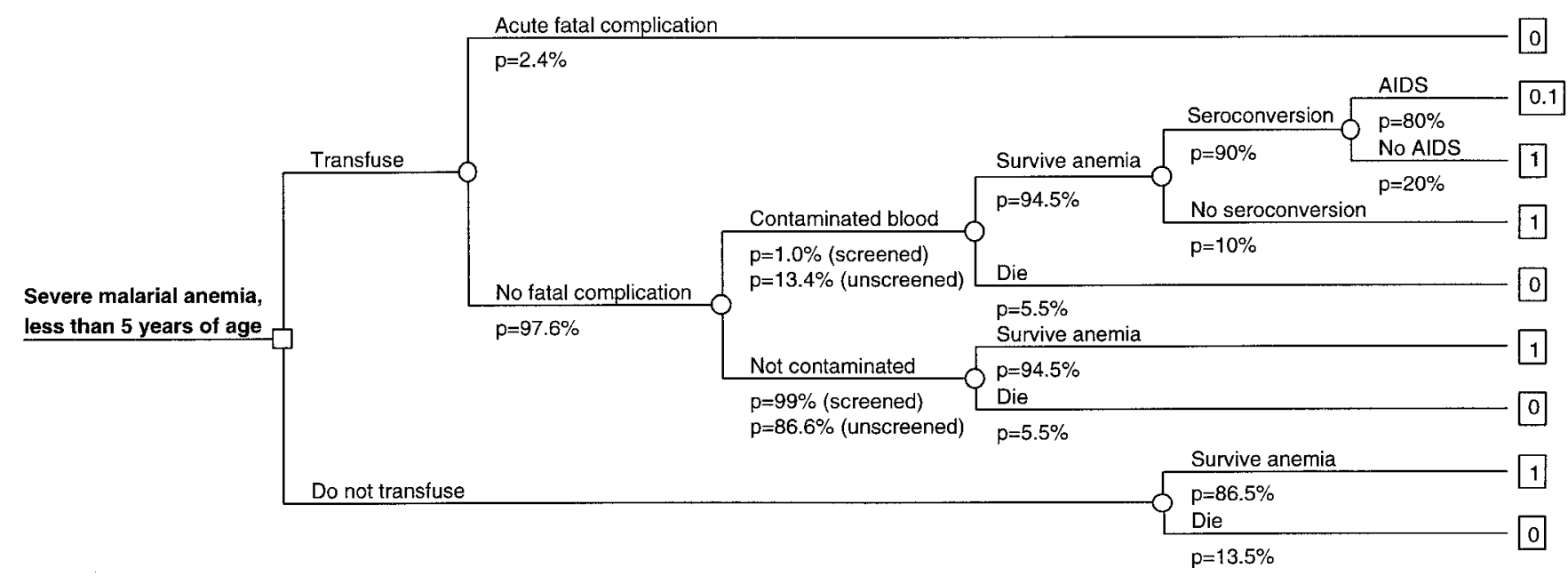

FIGURE 1. Transfusion decision tree. O represents a chance node, and $\square$ represents a decision node. p indicates the probability of occurrence of an event, as used in the base-case analysis. The numbers enclosed in square boxes at the terminal nodes are the valuations for the outcomes. AIDS $=$ acquired immunodeficiency syndrome.

tients had no HIV infection before treatment. Finally, we assumed that an effective antimalarial drug (e.g., quinine) had already been administered.

Model quantification. Probabilities of the occurrence of the different events in the decision analysis were based on published studies as identified with a MEDLINE search. Plausible ranges were defined for the probabilities, based on 95\% confidence intervals (CIs) or ranges found in the literature.

To determine when the benefits of transfusion outweighed the risks, we assumed that short-term mortality was the worst outcome (valued as 0 ), and survival without AIDS the best (valued as 1). Young children may develop AIDS within two years and $50 \%$ may have died 5 five years later. ${ }^{25}$ We therefore estimated that children with HIV and seroconversion to AIDS may have a life expectancy of approximately one-tenth the normal life-expectancy among African children. ${ }^{25}$ The outcome AIDS was valued as 0.1 , with a plausible range from 0 , when AIDS is considered as bad as shortterm mortality, to 0.2 , when AIDS is regarded as equivalent to one-fifth of normal survival.

A sensitivity analysis was performed to gain insight in the effects of variation in the estimates on the difference between survival with or without transfusion. Calculations were performed with the DATA 3.0 (TreeAge Software, Inc., Williamstown, MA) computer package.

TABLE 1

Severe malarial anemia mortality studies in Africa, 1990-1997

\begin{tabular}{|c|c|c|c|c|}
\hline First author, year & $\mathrm{N}$ & Deaths & $(\%)$ & $\begin{array}{c}\text { Transmission } \\
\text { pattern }\end{array}$ \\
\hline Lackritz, $1992^{7}$ & 684 & 125 & (18.3) & High \\
\hline Brewster, $1993^{18}$ & 1,050 & 101 & $(9.6)$ & Low \\
\hline Craighead, $1993^{26}$ & 155 & 15 & $(9.7)$ & Moderate \\
\hline Holzer, $1993^{27}$ & 105 & 3 & $(2.9)$ & Moderate \\
\hline Slutsker, $1994^{6}$ & 910 & 140 & $(15.3)$ & Moderate \\
\hline Marsh, $1995^{16}$ & 506 & 25 & $(4.9)$ & Low \\
\hline Zucker, $1996^{19}$ & 126 & 14 & (11.1) & High \\
\hline Newton, $1997^{28}$ & 46 & 4 & $(8.7)$ & Low \\
\hline Total & 3,582 & 427 & (11.9) & \\
\hline
\end{tabular}

\section{RESULTS}

A base-case patient was defined as a two-year-old boy with a hemoglobin level $<5 \mathrm{~g} / \mathrm{dL}$ and an acute $P$. falciparum malaria infection. The probabilities that were used as estimates for this base-case patient are shown in the decision tree (Figure 1).

Risk of in-hospital mortality with severe malarial anemia. We identified eight prospective hospital-based studies conducted in African settings that had reported mortality rates associated with severe malarial anemia between 1990 and $19976,7,16,18,19,26-28$ (Table 1 ). The average risk of mortality was $12 \%$, but the heterogeneity was significant $(P<0.001$, by chi-square test). When the studies were classified according to the local malaria transmission level, we observed that children residing in high transmission regions experienced the highest mortality rates (139 [17.2\%] of 810 died), followed by moderate and low transmission areas (158 [13.5\%] of 1,170 died and 130 [8.1\%] of 1,602 died, respectively). This distinction according to transmission pattern explained a large proportion of the heterogeneity between the studies. However, differences between studies from low and moderate transmission areas were still statistically significant $(P$ $<0.05$, by chi-square tests). Furthermore, the case mix in these studies included both children who received transfusions and others who did not. The actual mortality risk may thus be higher for those who were not transfused.

In any specific malaria-endemic region, individual risks of mortality may vary considerably depending on the clinical presentation of the patient. For instance, hospitalized children on the Kenyan coast, who had severe anemia with respiratory distress, impaired consciousness, or both experienced $16 \%, 8 \%$, and $36 \%$ mortality rates, respectively. ${ }^{16}$ The mortality rate used for the decision model was $13.5 \%$ for the base-case (intermediate transmission region), with a range from $3 \%$ to $30 \%$.

Transfusion benefits and risks. The beneficial effect of a transfusion on in-hospital mortality was derived from a study in western Kenya. The observed odds ratio for inhospital mortality, hereafter referred to as transfusion effec- 
TABLE 2

Probability estimates and plausible ranges used in the decision analysis*

\begin{tabular}{lcc}
\hline \multicolumn{1}{c}{ Variable } & $\begin{array}{c}\text { Baseline } \\
\text { estimate } \dagger\end{array}$ & $\begin{array}{c}\text { Plausible range } \\
(\%)\end{array}$ \\
\hline $\begin{array}{l}\text { Mortality from untreated anemia } \\
\quad \text { (moderate transmission level) }\end{array}$ & $13.5 \%$ & $3-30$ \\
Transfusion effectiveness (odds ratio) & 2.7 & $1.9-3.7 \ddagger$ \\
HIV contamination risk & & \\
$\quad$ Screened blood & $1.0 \%$ & $0.5-20$ \\
$\quad$ Unscreened blood & $13.4 \%$ & 9,29 \\
Probability of HIV seroconversion & $90 \%$ & $90-96$ \\
Probability of AIDS development & $80 \%$ & 30,31 \\
Acute fatal complications risk & $2.4 \%$ & 32 \\
\hline
\end{tabular}

* HIV = human immunodeficiency virus; AIDS = acquired immunodeficiency syndrome $\dagger$ Values used in calculations for the base-case.

$\$ 95 \%$ confidence intervals.

tiveness, was $2.7(95 \% \mathrm{CI}=1.9-3.7) .{ }^{7}$ This means that the mortality odds with transfusion was 2.7 times lower than the mortality odds without a transfusion.

When the transfused blood is unscreened, the probability that it is HIV contaminated depends on the seroprevalence levels among the donor population. The probability that a screened unit of blood is HIV contaminated has been estimated at between $0.5 \%$ and $1 \%$ in Cote d'Ivoire, where HIV prevalence among blood donors was $11 \% .{ }^{29}$ These risks were $2.1 \%$ for screened blood (Lackritz EM and others, unpublished data) and $13.4 \%$ for HIV prevalence ${ }^{9}$ in western $\mathrm{Ke}$ nya. The plausible range for the probability of receiving HIV contaminated blood was set as $0.5-20 \%$. The probability that seroconversion occurs after receipt of HIV-contaminated blood has been found to be $90 \%$ in the United States ${ }^{30}$ and $96 \%$ in Zaire. ${ }^{31}$ The probability of developing AIDS after seroconversion is approximately $80 \% .32$ A $2.4 \%$ incidence of fatal acute transfusion complications was observed among transfused children in Kinshasa, Zaire. ${ }^{11}$ Table 2 summarizes the estimates and plausible ranges used in the decision analysis.

Transfusion decisions. When we evaluated the decision tree for the base-case patient, transfusion with screened blood led to a $7.7 \%$ risk of in-hospital mortality and $0.7 \%$ risk of acquiring AIDS, compared with a $13.5 \%$ mortality without transfusion. The survival benefit was $5.2 \%$. If the blood was not screened, we expected an $8.9 \%$ risk of acquiring AIDS, and the survival with transfusion was lower than without $(84.3 \%$ versus $86.5 \%)$. We further varied each estimate over its plausible range. Three fac- tors strongly influenced the benefit of transfusion: the blood contamination risk, the baseline mortality risk, and the transfusion effectiveness. For the base case, transfusion was the optimal decision provided that either the blood contamination risk was $<10 \%$, the transfusion-reduced mortality had an odds ratio $>1.3$, or the baseline risk of mortality was $>5 \%$. A minimal influence on the transfusion decision was noted for the valuation of AIDS, or the risks of fatal complications, seroconversion to HIV infection, or development of AIDS; for these estimates, variation over the plausible ranges exerted a maximal survival difference of $1 \%$.

We examined eight possible scenarios based on all combinations of low and high values of the three most important factors. Table 3 shows that a transfusion is clearly beneficial if the baseline mortality risk is high and the blood contamination risks are low. A net benefit is also expected if all three factors are on their high values (scenario 4). In the other scenarios, transfusion leads to a lower survival. For instance, transfusion is not indicated for a severely anemic child with a low risk of mortality, especially if only unscreened blood is available.

Finally, we varied the risks of blood contamination, the baseline mortality risk and the transfusion effectiveness in a three-way sensitivity analysis (Figure 2). The graph shows that for three levels of transfusion effectiveness, blood transfusion leads to a larger benefit in survival if the mortality risk is higher and the contamination risk is lower. Also, at a constant blood contamination risk, the threshold (mortality risk) above which one would trans-

TABLE 3

Eight possible scenarios based on combinations of low $(\mathrm{L})$ and high $(\mathrm{H})$ plausible values for the HIV contamination risk in transfused blood, the anemia mortality risk, and the transfusion effectiveness*

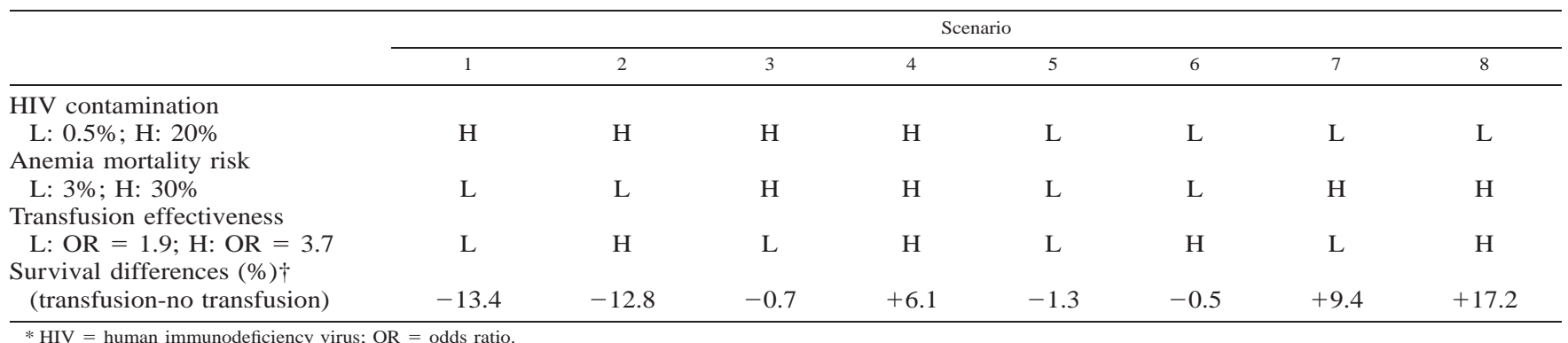

$\dagger$ Positive values indicate that transfusion is the preferred strategy. 


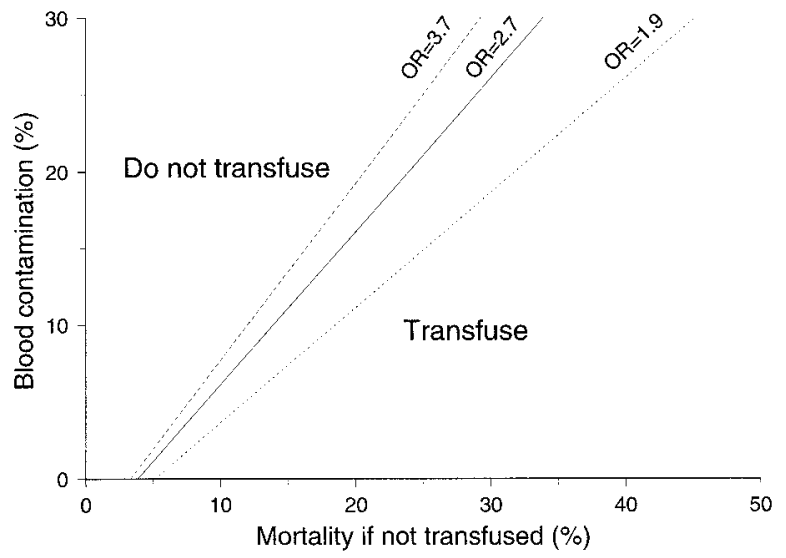

FIGURE 2. Three-way sensitivity analysis for survival with or without blood transfusion. The lines represent the combinations of mortality risk and human immunodeficiency virus contamination risk where survival with or without transfusion is equal for three estimates of the effectiveness of transfusion (mortality odds ratios $[\mathrm{ORs}]=1.9,2.7$, and 3.7). To the right of each line, survival with a transfusion is higher than without one.

fuse decreases with increasing transfusion effectiveness. Overall, we note that transfusion does not carry a survival benefit when HIV contamination risk is high.

\section{DISCUSSION}

In this analysis we examined when it is beneficial to administer a blood transfusion to African children with severe malarial anemia. Three factors were found to have a major impact on this decision: the risk of mortality without a transfusion, HIV contamination risk of the blood products, and the effectiveness of a transfusion in reducing mortality.

We estimated the average risk of mortality with severe malarial anemia to be approximately $12 \%$, but this figure varied considerably across reported studies. The heterogeneity between studies was partly explained by differences in the intensity of malaria transmission patterns. ${ }^{21,33}$ Furthermore, the mortality risk may vary depending on several clinical characteristics, such as respiratory distress, impaired consciousness, age, hemoglobin level, parasite density, and comorbidity. ${ }^{7,16-22}$ Transfusion was not beneficial when the patient's risk of mortality without a transfusion was $<5 \%$. Because most deaths among severely anemic children tend to occur within $12 \mathrm{hr}$ of admission, it is important to identify those patients at high risk timely to allow for early intervention. When transfusion is delayed, the child already survived the immediate period and the remaining mortality risk will be lower.

The effectiveness of blood transfusion has been found to be higher with a higher patient-specific mortality risk and an early administration of the transfusion. ${ }^{7}$ Decision making shortly after admission of the patient is thus required. This may best be achieved with the availability of a blood bank with screened blood. In clinical practice, the estimate of a reduction of the mortality with an odds ratio of 2.7 may be a starting point, while the impact of other assumptions of effectiveness can be explored in Figure 2. Randomized clinical trials are desired to provide further evidence on the benefits and risks of transfusion. Such studies might be consid- ered for children with a moderate mortality risk, e.g., approximately $5-10 \%$, since the benefit of a transfusion is unclear for these children. However, the definition of this risk group may be difficult, and the conclusions might have limited generalizability.

As expected, the likelihood of blood contamination with the HIV-1 virus had a crucial influence on the benefit of a transfusion. African children less than five years of age with acute malarial anemia or sickle cell anemia are the most frequent recipients of blood transfusions. For instance, at one hospital in western Kenya, children received $67 \%$ of all the administered transfusions. ${ }^{7}$ With the increasing prevalence of pediatric malaria-associated anemia, blood screening is therefore a priority child survival issue. ${ }^{34}$ Most African countries have a policy of pretransfusional blood screening, which may, however, be difficult to achieve in practice due to logistic and technical limitations. It has been observed that HIV contamination may be up to five times higher among units donated for replacement or by family members compared with banked blood from volunteer donors. Moreover, only banked blood will be available early during hospitalization, when transfusion may be most effective to increase survival. ${ }^{7,9}$ Because of the increasing HIV prevalence among blood donors in most African settings, safe blood has become a scarce and costly resource. It was estimated to cost $\$ 14$ to remove one HIV-contaminated unit by blood screening in Kenya (Lackritz EM and others, unpublished data). Our analysis shows that using screened blood implies a substantial benefit compared with using unscreened blood (survival $+5 \%$ versus $-2 \%$ ). Therefore, it may be expected that the cost-effectiveness of blood screening is favorable in the context of transfusion of severe malaria-related anemia in African children. Our analysis indicates that unscreened blood should only be given in life-saving situations. This finding supports current transfusion policies that generally discourage the use of unscreened blood. We recommend continued intensification of efforts to ensure a safe blood supply by screening and recruitment of low-risk donors.

Although this analysis was undertaken on a number of assumptions, it has enabled us to study important considerations relating to when a transfusion may be beneficial. The methodology that we adopted can be used to study other transfusion-transmissible infections, such as hepatitis B and C. The results shown in Figure 2 may be applicable in all settings in which malarial anemia and transfusion-transmitted HIV-1 are important health problems. We note, however, that the specific probability estimates used in our analysis mainly originate from anglophone Africa, which may not be representative for all of Africa. Furthermore, this analysis did not consider the many nonclinical factors that trigger and influence the clinical decision to order transfusions. These may include the health-care provider's perception and tolerance of risk, peer-pressure, and the availability of blood. ${ }^{35,36}$

In conclusion, our study has provided a quantitative insight in the relevant considerations for making transfusion decisions in African children with severe malaria-related anemia. This insight may support the development of clinical transfusion policies at a local level such that transfusions be limited to situations where they are likely to be beneficial. 
Acknowledgments: This research has been carried out as part of the MSc program in Clinical Epidemiology of the Netherlands Institute for Health Sciences, Erasmus University, Rotterdam, The Netherlands. We acknowledge the contributions made by Frithjofna Abbink, Carina van Vliet, and Kitty van der Ploeg to this study. We appreciate comments by Dr. Maribel Salas on earlier drafts of this report.

Authors' addresses: Charles O. Obonyo and Aggrey J. Oloo, Vector Biology and Control Research Centre, Kenya Medical Research Institute, PO Box 1578, Kisumu, Kenya. Ewout W. Steyerberg and J. Dik F. Habbema, Center for Clinical Decision Sciences, Department of Public Health, Erasmus University Medical School, PO Box 1738, 3000 DR Rotterdam, The Netherlands.

\section{REFERENCES}

1. AuBuchon JP, 1996. The role of decision analysis in transfusion medicine. Vox Sang 71: 1-5.

2. Greenberg AE, Nguyen-Dinh P, Mann JM, Kabote N, Colebunders RL, Francis H, Quinn TC, Baudoux P, Lyamba B, Davachi F, Roberts JM, Kabeya N, Curran JW, Campbell CC, 1988. The association between malaria, blood transfusion and HIV seropositivity in a pediatric population in Kinshasa, Zaire. JAMA 259: 545-549.

3. Bloland PB, Lackritz EM, Kazembe PN, Were JB, Steketee R, Campbell CC, 1993. Beyond chloroquine, implications of drug resistance for evaluating malaria therapy and treatment policy in Africa. $J$ Infect Dis 167: 932-937.

4. Campbell CC, 1991. Challenges facing antimalarial therapy in Africa. J Infect Dis 163: 1207-1211.

5. Hedberg K, Shaffer N, Davachi F, Hightower A, Lyamba B, Paluku KM, Nguyen-Dinh, P, Breman JG, 1993. Plasmodium falciparum-associated anemia in children at a large urban hospital in Zaire. Am J Trop Med Hyg 48: 365-371.

6. Slutsker L, Taylor TE, Wirima JJ, Steketee RW, 1994. In-hospital morbidity and mortality due to malaria-associated severe anemia in two areas of Malawi with different patterns of malaria infection. Trans R Soc Trop Med Hyg 88: 548-551.

7. Lackritz EM, Campbell CC, Ruebush TK II, Hightower AW, Wakube W, Steketee RW, Were JB, 1992. Effect of blood transfusion on survival among children in a Kenyan hospital. Lancet 340: 524-528.

8. Dorward JA, Knowles JK, Dorward IM, 1989. Treatment of severe anaemia in children in a rural hospital. Trop Doctor 19: $155-158$.

9. Lackritz EM, Ruebush TK II, Zucker JR, Adungosi JE, Were JB, Campbell CC, 1993. Blood transfusion practices and blood banking services in a Kenyan hospital. AIDS 7: 995999.

10. Mhalu FS, Ryder RW, 1988. Blood transfusion and AIDS in the tropics. Baillieres Clin Trop Med Commun Dis 3: 157166.

11. Jager H, N'Galy B, Perriens JP, Nseka K, Davachi F, Kabeya CM, Rauhaus G, Peyerl G, Ryder RW, Rehle T, 1990. Prevention of transfusion-associated HIV transmission in Kinshasa, Zaire: HIV screening is not enough. AIDS 4: 571-574.

12. Gibbs WN, Corcoran P, 1994. Improving safety of blood transfusion in developing countries. Vox Sang 69: 61-66.

13. Ryder RW, 1992. Difficulties associated with providing an HIVfree blood supply in tropical Africa. AIDS 6: 1395-1397.

14. N'tita I, Mulanga K, Dulat C, Lusamba D, Rehle T, Korte R, Jager H, 1991. Risk of transfusion-associated HIV transmission in Kinshasa, Zaire. AIDS 5: 437-439.

15. Ward JW, Holmberg SD, Allen JR, Cohn DL, Critchley SE, Kleinman SH, Lenes BA, Ravenholt O, Davis JR, Quinn MG, Jaffe HW, 1988. Transmission of human immunodeficiency virus (HIV) by blood transfusions screened as negative for HIV antibody. N Engl J Med 318: 473-478.

16. Marsh K, Forster D, Waruiru C, Mwangi I, Winstanley M, Marsh V, Newton C, Winstanley P, Warn P, Peshu N, Pasvol
G, Snow R, 1995. Indicators of life-threatening malaria in African children. N Engl J Med 332: 1399-1404.

17. Molyneux ME, Taylor TE, Wirima JJ, Borgstein A, 1989. Clinical features and prognostic indicators in paediatric cerebral malaria: a study of 131 comatose Malawian children. $Q \mathrm{~J} \mathrm{Med}$ 71: 441-459.

18. Brewster DR, Greenwood BM, 1993. Seasonality of paediatric diseases in the Gambia, West Africa. Ann Trop Paediatr 13: 133-146.

19. Zucker JR, Lackritz EM, Ruebush TK II, Hightower AW, Adungosi JE, Were JB, Metchock B, Patrick E, Campbell CC, 1996. Childhood mortality during and after hospitalization in western Kenya: effect of malaria treatment regimens. Am $J$ Trop Med Hyg 55: 655-660.

20. Commey JO, Dekyem P, 1995. Childhood deaths from anemia in Accra, Ghana. West Afr J Med 14: 101-104.

21. Snow RW, Bastos de Azevedo I, Lowe BS, Kabiru EW, Nevill CG, Mwankusye S, Kassiga G, Marsh K, Teuscher T, 1994. Severe childhood malaria in two areas of markedly different falciparum transmission in east Africa. Acta Trop 57: 289300.

22. Phillips RE, Pasvol G, 1992. Anaemia of Plasmodium falciparum malaria. Ballieres Clin Haematol 5: 315-330.

23. Weinstein MC, Fineberg HV, 1980. Clinical Decision Analysis. Philadelphia: WB Saunders.

24. Heymann SJ, Brewer TF, 1993. The infectious risks of transfusions in the United States: a decision-analytic approach. Am J Infect Control 21: 174-182.

25. Lepage P, Hitimana DG, 1991. Natural history and clinical presentation of HIV-I infection in children. AIDS (suppl 1): S117-S125.

26. Craighead IB, Knowles JK, 1993. Prevention of transfusionassociated HIV transmission with the use of a transfusion protocol for under five. Trop Doctor 23: 59-61.

27. Holzer BR, Egger M, Teuscher T, Koch S, Mboya DM, Smith DG, 1993. Childhood anemia in Africa; to transfuse or not transfuse? Acta Trop 55: 47-51.

28. Newton CRJC, Warn PA, Winstanley PA, Peshu N, Snow RW, Pasvol G, Marsh K, 1997. Severe anaemia in children living in a malaria endemic area of Kenya. Trop Med Int Health 2: $165-178$.

29. Savarit D, De Cock KM, Schutz R, Konate S, Lackritz E, Bondurand A, 1992. Risk of HIV infection from transfusion with blood negative for HIV antibody in a west African city. $\mathrm{Br}$ Med J 305: 498-502.

30. Donegan E, Stuart M, Niland JC, Sacks HS, Azen SP, Dietrich SL, Faucett C, Fletcher MA, Kleinman SH, Operskalski EA, Perkins HA, Pindyck J, Schiff ER, Stites DP, Tomasulo PA, Mosley JW, and the Transfusion Safety Group, 1990. Infection with human immunodeficiency virus type I (HIV-1) among recipients of antibody-positive blood donations. Ann Intern Med 113: 733-739.

31. Colebunders R, Ryder R, Francis H, Nekwei W, Bahwe Y, Lebughe I, Ndilu M, Vercauteren G, Nseka K, Perriens J, Van der Stuyft P, Quinn TC, Piot P, 1991. Seroconversion rate, mortality, and clinical manifestations associated with the receipt of a human immunodeficiency virus-infected blood transfusion in Kinshasa, Zaire. J Infect Dis 164: 450-456.

32. Heymann SJ, Brewer TF, 1992. The problem of transfusionassociated acquired immuno-deficiency syndrome in Africa: a quantitative approach. Am J Infect Control 20: 256-262.

33. Greenwood BM, Marsh K, Snow R, 1991. Why do some African children develop severe malaria? Parasitol Today 7: 277-281.

34. Foster S, Buvé A, 1995. Benefits of HIV screening of blood transfusions in Zambia. Lancet 345: 225-227.

35. Brown RL, Brown RL, Edwards JA, Nutz JF, 1992. Variation in a faculty's decisions to transfuse; implications for modifying blood product utilization. Med Care 30: 1083-1096.

36. Scatz-Salem SR, Avorn J, Soumerai SB, 1990. Influence of clinical knowledge, organizational context and practice style on transfusion decision making. JAMA 264: 471-475. 